OPEN ACCESS

Edited by:

Per T. Sangild,

University of Copenhagen, Denmark

Reviewed by:

Egídio Torrado,

University of Minho, Portugal

Gunnveig Grødeland,

University of Oslo, Norway

*Correspondence:

Elizabeth Ann Bonney

elizabeth.bonney@med.uvm.edu

Specialty section:

This article was submitted to

Vaccines and Molecular Therapeutics,

a section of the journal

Frontiers in Immunology

Received: 02 June 2020 Accepted: 05 October 2020 Published: 28 October 2020

Citation:

Bonney EA, Krebs K, Kim J, Prakash $K$, Torrance BL, Haynes $L$ and Rincon M (2020) Protective Intranasal Immunization Against Influenza Virus in Infant Mice Is Dependent on IL-6.

Front. Immunol. 11:568978. doi: 10.3389/fimmu.2020.568978

\section{Protective Intranasal Immunization Against Influenza Virus in Infant Mice Is Dependent on IL-6}

\author{
Elizabeth Ann Bonney ${ }^{1 *}$, Kendall Krebs ${ }^{1}$, Jihye Kim ${ }^{2}$, Kirtika Prakash $^{1}$, \\ Blake L. Torrance ${ }^{3}$, Laura Haynes ${ }^{3}$ and Mercedes Rincon ${ }^{4,5}$ \\ 1 Department of Obstetrics, Gynecology and Reproductive Sciences, Lamer College of Medicine, University of Vermont, Burlington, \\ VT, United States, ${ }^{2}$ Division of Medical Oncology, Department of Medicine, University of Colorado, Anschutz Medical Campus, \\ Aurora, CO, United States, ${ }^{3}$ Department of Immunology, University of Connecticut Center on Aging, Farmington, CT, United States, \\ ${ }^{4}$ Division of Immunobiology, Department of Medicine, Larner College of Medicine, University of Vermont, Burlington, VT, United \\ States, ${ }^{5}$ Department of Immunology and Microbiology, University of Colorado Anschutz Medical Campus, Aurora, CO, United \\ States
}

Respiratory diseases adversely affect infants and are the focus of efforts to develop vaccinations and other modalities to prevent disease. The infant immune system differs from that of older children and adults in many ways that are as yet ill understood. We have used a C57BL/6 mouse model of infection with a laboratory- adapted strain of influenza (PR8) to delineate the importance of the cytokine IL-6 in the innate response to primary infection and in the development of protective immunity in adult mice. Herein, we used this same model in infant (14 days of age) mice to determine the effect of IL-6 deficiency. Infant wild type mice are more susceptible than older mice to infection, similar to the findings in humans. IL-6 is expressed in the lung in the early response to PR8 infection. While intramuscular immunization does not protect against lethal challenge, intranasal administration of heat inactivated virus is protective and correlates with expression of IL-6 in the lung, activation of lung CD8 cells, and development of an influenza-specific antibody response. In IL-6 deficient mice, this response is abrogated, and deficient mice are not protected against lethal challenge. These studies support the importance of the role of the tissue environment in infant immunity, and further suggest that IL-6 may be helpful in the generation of protective immune responses in infants.

Keywords: infant, influenza, mouse, vaccination, lung

\section{INTRODUCTION}

Respiratory tract inflammatory and infectious disease in infant children represents a significant burden to the healthcare system $(1,2)$. A particular example is influenza virus infection, for which there is evidence of increased incidence of disease severity in infants, as compared to adults in humans and animal models $(3,4)$. Classic theory suggests that adaptive immunity, predominantly the $\mathrm{T}$ cell immune response, is altered in infants in order to support self-tolerance, maternal 
tolerance, and tolerance to new developmental or environmental antigen (5-7). More recent thinking about infant immunity considers the possibility of distinct populations of $\mathrm{T}$ cells present in the infant, but not in the adult, that display a "tolerance" phenotype (8). Alternative models suggest that $\mathrm{T}$ cells in neonates/infants are not inherently tolerant, but that tissue specific signals can affect $\mathrm{T}$ cell activation and effector function by limiting or supporting the access to productive antigenic signals (9-17).

The role of the tissue environment (independently of the immune cells) in which specific immune responses occur could also be an important factor that affects the immune response in neonates/infants $(10,18)$. Specifically, for influenza virus and other respiratory infections, the epithelium of the respiratory tract provides a unique environment that can tune adaptive immune responses. Lung epithelial cells are a major source of type I IFN which plays a critical role in antiviral responses (19). In addition, lung epithelial cells can produce a variety of inflammatory cytokines (e.g., IL-6) that can influence the course of T cell responses (19). Lung epithelial cells in infants differ from those in adults in humans (20-23) and animals (24). The potential effect of lung epithelial cells in the immune responses of infants is a recent area of inquiry, and could be a significant determining factor in their apparent altered immune response during influenza virus infection.

Although intramuscular administration of a polyvalent formulation of chemically inactivated or disrupted influenza virus is the most common type of vaccine for seasonal influenza both in adults and children (25), intranasal administration of a live attenuated influenza virus (LAIV) in the respiratory tract is also utilized as a method of vaccination (26). However, this formulation is restricted to children more than 2 years of age and adults $(27,28)$ because of reports of increased risk of reactive airway disease (29, 30) and increased concern over the presence of underlying asthma in young vaccine recipients $(31,32)$. The factors underlying the relatively lower effectiveness of recent intranasal vaccination with live attenuated virus compared to intramuscular administration of inactivated virus in some flu seasons (33-35) remain incompletely understood. Together, these issues elevate the question of whether there might be novel specific mechanisms or alternative approaches to enhance influenza vaccination in the very young. Similar to the response to respiratory virus infection, lung epithelium could also play a key role in determining the type and strength of the immune response that intranasal immunizations can trigger.

Interleukin 6 (IL-6) is a member of a family of cytokines that play an important role in both innate and adaptive immune responses (36). In addition, IL-6 is important in the processes of tissue regeneration and inhibition of apoptosis (37). IL-6 is produced by innate immune cells such macrophages, but it is also produced by a variety of cell types (e.g. epithelial cells, endothelial cells, astrocytes) upon exposure to insults (36). For example, we and others have shown that IL- 6 is produced by lung epithelial cells in response to viral infection or allergens (38, 39). In adult mice, IL-6 can enhance T-cell mediated antibody response against i.m. influenza immunization (40) and there is evidence supporting the importance of IL- 6 in the early response to influenza infection (41). However, the specific importance of IL-6 in infant immunity to influenza is less clear (42).

Here, we have performed gene expression profiling studies comparing lung epithelial cells from infant and adult mice and the results have revealed a compromised expression of IL-6 and related signaling pathways in infant lung epithelial cells. Using a heat-inactivated influenza virus, as a surrogate for current attenuated virus formulations, we show that i.m. administration in infant mice does not provide protection, while intranasal administration does. However, such protection induced by i.n. immunization is dependent on IL-6. Thus, these studies underline the relevance of the tissue environment for the efficacy of vaccinations in infants, and they bring to light potential mechanisms related to differences in infant and adult lung epithelium that could influence the efficacy of immunizations. Our results could be relevant for future improvement of vaccines for young children.

\section{MATERIAL AND METHODS}

\section{Mice}

C57BL/6J (wild type, WT obtained from Jackson Laboratory) or IL-6 KO mice (43) were housed under specific pathogen free, AAALAC-approved conditions using a 12-h light cycle and were given food (normal Chow) and water ad libitum. IL-6 KO mice used in these studies were backcrossed over 12 generation with C57Bl/6J. Females of both strains and 8-24 weeks of age mated freely with same strain males and littered without interference. Thirteen to fourteen days after birth, while still nursing, mothers and infant mice moved to a biosafety room, where they acclimated for $12-24 \mathrm{~h}$ before infection or immunization of the pups.

\section{Influenza Virus Infections and Immunizations}

These studies utilized Puerto Rico A/PR/8/34 H1N1 influenza A (PR8) (41). Intranasal and intramuscular immunizations utilized heat inactivated PR8 influenza virus (iPR8). Inactivation was performed by incubation at 56 degrees for $30 \mathrm{~min}$. This method of inactivation allows production of a virus that enters cells, since it does not fully denature the HA protein of the virus, but which does not replicate since the virus polymerase is made inactive at this and lower temperatures $(44,45)$. iPR8 $\left(5 \times 10^{5}\right.$ EIU) in $15-$ 20 or $50 \mu \mathrm{l}$ PBS was used for intranasal or intramuscular immunizations, respectively. The average weight was 5.9+/$0.15 \mathrm{~g}$ at $14-16$ days of life and this was not statistically different from any experimental group (Supplementary Figure S1). In two experimental cohorts, mice were euthanized three weeks after immunization to collect tissues for in vitro examination of the immune response to immunization.

For lethal challenge in juvenile mice (day 35-48 of life) we used $6 \times 10^{3}$ EIU PR8 given intranasally in $50 \mu \mathrm{l}$ of PBS. For the batch of PR8 virus used in these studies, $10^{4}$ EIU corresponded to 2 LD50 when initially tested in adult mice (8-10 weeks of age). For juvenile mice between $30-45$ days of age, we found 
$6 \times 10^{3}$ EIU PR8, could be equivalent to the 2 LD50 in adult mice (Supplementary Table I). Female mice weighed 16 \pm 1 gram and males weighed $20 \pm 1$ gram at the time of challenge. Mice underwent inhalant anesthesia $(1.5 \mathrm{~L} / \mathrm{min} \mathrm{O} 2,2 \%$ isoflurane $)$ in a chamber connected to an isoflurane vaporizer to receive intranasal immunizations or infections with live virus. Infected mice were weighed every $24-48 \mathrm{~h}$. Mice reaching below $70 \%$ of starting weight were euthanized consistent with stipulations of our animal use protocol (University of Vermont IACUC\# 13-029). Therefore "survival" in these studies indicates mice who did not fall below this threshold. Though males of each group at challenge were heavier than same strain females, the weights of WT and IL6KO females and those of WT and IL6KO males were comparable (Supplementary Figure S2). To account for male and female weight differences, males were challenged with higher doses. Supplementary Figure $\mathbf{S} 3$ shows a representative sample of the weight of WT females who were challenged with virus.

\section{Determination of Influenza Viral Load in Tissues}

Lungs from assayed mice were freshly harvested and frozen in liquid nitrogen. RNA was isolated from whole lung tissue homogenized in TRIzol reagent (Invitrogen Life Technologies). cDNA was synthesized using iScript cDNA synthesis kit (BioRad Laboratories), using the manufacturer's protocol. Viral loads in harvested whole lungs were determined by real-time RT-PCR for the PR8 viral acid polymerase (PA) gene by comparison to a standard titration of viral PA copies run on the same PCR assay, with 20ng of cDNA used per reaction. The following primers and probe were used to amplify and quantitate the PR8 PA gene: forward primer, 5'-CGGTCCAAATTCCTGCTGA-3'; reverse primer, 5' - CATTGGGTTCCTTCCATCCA-3'; probe, 5'-6FAM-CCAAGTCATGAAGGAGAGGGAATACCGCT-3' (Integrated DNA Technologies) (41).

\section{Cytokine Gene Expression}

In alternate RNA isolation protocols, the Qiagen RNeasy Mini kit (PN 74104) was utilized as recommended by the manufacturer. cDNA was synthesized as above. Relative mRNA levels were determined by qRT-PCR using Assays-on-Demand TaqMan Gene Expression Assays (FAM-MGB, ThermoFisher Scientific https:// www.thermofisher.com) for IL-6(Mm00446190), CCL2 (Mm00441242), $\gamma \mathrm{IFN}(\mathrm{Mm01168134),} \mathrm{IL-10(Mm01288386),} \mathrm{TNF}$ (Mm00443258), TGFB1 (Mm01337605), and Beta-2 microglobulin (Mm00437762). Values reported are those obtained after normalization to $\beta 2$-microglobulin and analyzed by the comparative delta CT method. In addition, serum cytokines were quantified using a Luminex ${ }^{\circledR} \mathrm{xMAP}^{\circledR}$ multiplex platform, combined with a customized Milliplex ${ }^{\mathrm{TM}}$ mouse chemokine/cytokine panel from Millipore ${ }^{\mathrm{TM}}$.

\section{Analysis of Anti-Influenza Virus Specific Antibodies in Serum by ELISA}

Influenza-specific antibody levels in serum samples were determined by ELISA, as previously described (40). ELISA plates were coated with inactive influenza PR8 virus $\left(10^{7} \mathrm{EIU} / \mathrm{ml}\right)$ in sodium bicarbonate buffer, washed, blocked (1\% BSA/PBS solution) and incubated with 2-fold serial dilutions of serum overnight. Plates were washed and incubated with HRP-conjugated goat anti-mouse total IgG (SouthernBiotech) for $45 \mathrm{~min}$ at room temperature. Plates were then washed and developed using TMB Sureblue substrate and development was stopped with TMB stop solution (ThermoFisher). Plates were read at $450 \mathrm{~nm}$ in a plate reader.

\section{Flow Cytometry Analyses of Lungs}

Whole lungs harvested from immunized mice were used to prepare single cell suspensions using the gentle MACS $^{\mathrm{TM}}$ (Miltenyi Biotech) tissue dissociation system. Red cells were removed with Geyes lysis medium and the resulting cell suspensions were washed in Iscove's Modified Dulbecco's medium with 5\% FBS. Cell suspensions were stained with antibodies to CD45 (CD45.2, clone 104, PerCP-Cy ${ }^{\mathrm{TM}}$ 5.5), CD8(CD8 $\alpha$ clone 53-6.7, Pacific Blue ${ }^{\mathrm{TM}}$ ) CD4 (clone GK1.5, R-phycoerythrin) and CD44 (clone IM7 fluorescein isothiocyanate) and were run on an LSRII (BD Biosciences). The gating scheme for these studies is shown in Supplementary Figure S5.

\section{Lung Epithelial Cell Gene Expression Analysis}

Four samples, each consisting of pooled epithelial cells from the lungs of three male pups or three female pups aged 14 days (total eight pups samples), or epithelial cells from four individual male and four female adult (eight weeks) lungs (eight adult samples total) were used to isolate RNA and examined by array transcriptome profiling. Mice were euthanized by cervical dislocation. Lungs were removed under sterile conditions into $1 \mathrm{X}$ PBS and cut up into very small pieces. Tissues were transferred to MACS C-tubes (purple tubes) for homogenization using the gentle MACS $^{\mathrm{TM}}$ (Miltenyi Biotech) tissue dissociation system. Red cells were removed by treatment with Geye's solution, and this was followed by resuspension in DMEM/F12+ 5\% FBS. The resulting cell suspension was incubated in a cell culture plate at 37C degrees and 5\% CO2. Afterwards, nonadherent epithelial cells were removed by slowly rocking the plate back and forth and gently removing the supernatant. This was centrifuged, resuspended and washed in MACS ${ }^{\mathrm{TM}}$ buffer and then incubated with anti-CD45 (Miltenyi) beads to remove CD45 + cells by passing the incubated solution on a magnetized LS column (Miltenyi). Purity was checked by flow cytometry for CD45 (less than $5 \%$ ) and histochemical identification of keratin + cells. Transcriptome profiling was done using the Affymextrix GeneChip system (Mouse Gene 2.0 ST Array). Chip quality was verified, and scan data was analyzed using RMA (46). Analysis of array data was performed using Partek Genomics Suite ${ }^{\circledR}$ 6.6. Beta Analysis. We report comparisons between groups using the number of probe sets that pass an FDR of 0.05 , or a binary filter $(\mathrm{p}<0.05$ and $2 \mathrm{x}$ fold change).

\section{Functional Analysis}

We used GSEA (Gene Set Enrichment Analysis) and pathway analysis, an approach that offers an unbiased global search for genes that are coordinately regulated in predefined pathways (47) rather than interrogating expression differences of single genes. Gene set analysis was performed using the GSEA software (48) 
version 4.0.3 obtained from https://www.gsea-msigdb.org/gsea/ downloads.jsp. The gene sets database was compiled from the Kyoto Encyclopedia of Genes and Genomes (KEGG) database http://www.kegg.jp/ (47). The KEGG gene sets database contains 210 mouse pathways that include metabolism, genetic information processing, environmental information processing, cellular processes, and human diseases. One hundred eight-eight gene sets passed the gene set size filter criteria ( $\mathrm{min}, 10$; max, 500). $P$ values for the gene sets were computed by permuting the gene sets 1,000 times in this study.

\section{Additional Statistical Analysis}

Viral load, cytokine levels, and antibody level were compared using one-way ANOVA or nonparametric analysis as appropriate. Due to small numbers, most normality testing used the Shapiro-Wilk test. Survival analysis with threshold being weight below 70\% of starting (point at which we were compelled to euthanize mice) was performed using the log-rank (Mantel-Cox) test. Data shown represents survival analysis of combined data of percentage of initial weight over time from challenge from over 8 cohorts of mice that received immunization and or challenge as indicated. Means \pm SEM or Median with range are reported, depending on the hypothesis test used. For hypothesis testing, significance was set at $\mathrm{p}<0.05$.

\section{RESULTS}

\section{High Mortality to Sublethal Dose of Influenza Virus in Infant Mice}

Similar to humans, infection with a sublethal dose of influenza virus in adult mice leads through a period of sickness during the peak of virus replication, and eventual recovery upon virus clearance from the lung. Several lines of evidence suggest that children less than five years of age are more susceptible to seasonal influenza virus infection $(3,49)$ and this led us to first investigate the age-related susceptibility of infant mice to influenza. We performed viral infection in mice 10-44 days old with different doses of PR8 influenza virus. Because the size of the lungs is determined by the body size of the mouse, we used different doses to normalize by weight. As we have shown (41), nearly all young adult ( 5 weeks of age) mice survived infection with a sublethal dose of influenza (Figure 1 and Supplementary Table 1). When we infected 24-dayold mice with same viral dose/weight ratio, we observed a small fraction of mortality (Figure 1 and Supplementary Table 1). In contrast, infection of 10 day or 14-16-day-old mice with a comparative viral dose/weight ratio caused $\sim 75 \%$ mortality (Figure 1 and Supplementary Table 1). Thus, when corrected for body size, infants are highly susceptible to an otherwise sublethal dose of PR8 virus infection.

\section{Intranasal, but Not Intramuscular, Immunization With Inactive Influenza Virus Provides Protection in Infant Mice}

Because very young children, like infant mice examined above, are more susceptible to death with influenza virus, there has been pressure to identify the most appropriate and efficacious way to immunize them against influenza. However, existing practical, clinical, and biologic limitations on the type of influenza vaccine that is currently provided to very young children make this issue a subject of intense investigation. We therefore used our mouse model above to determine a route and format of immunization that could protect infant mice against lethal infection. Because most children only receive an intramuscular injection of influenza vaccine, often with minor benefit (50), we compared

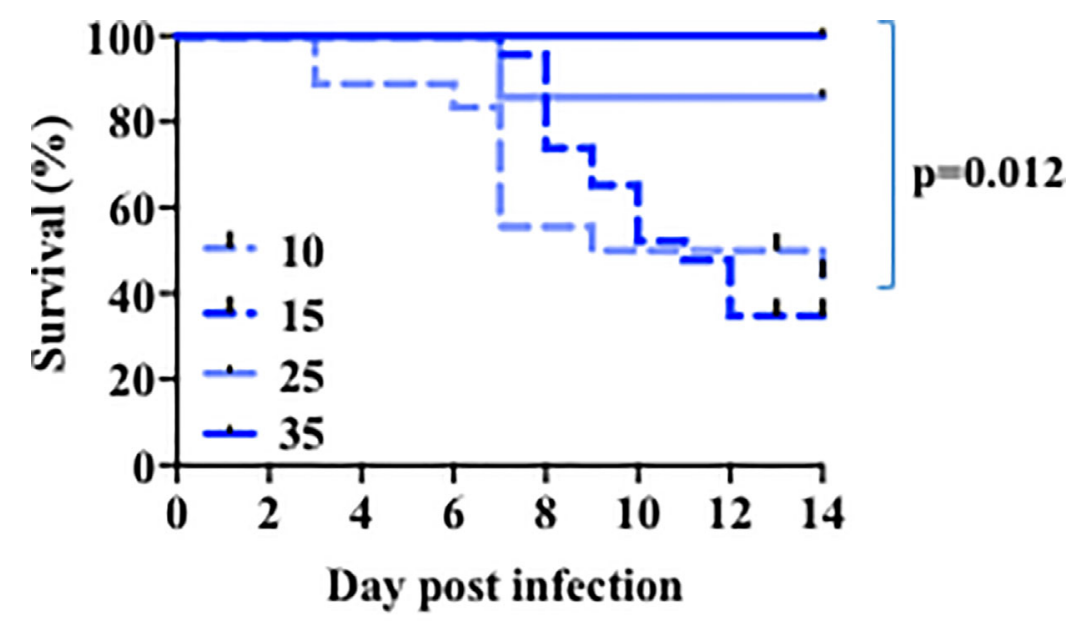

FIGURE 1 | Age-related susceptibility to a sublethal dose of PR8 influenza virus. The 10 days-old mice group $(n=18)$ received 140-200 EIU ( 30 ElU per gram of weight) of live PR8 influenza virus, the 15 days-old mice group ( $n=23)$ received 200 EIU ( 30 ElU per gram of weight), the 25 days-old mice group ( $n=7)$ received 300-900 EIU ( 30 EIU per gram of weight), the 35 days-old mice group $(n=6)$ received 3000 EIU ( 200 EIU per gram of weight). Survival was followed. Solid lines, older mice. Dotted lines, infant mice. $p$ value, comparison of curves for day 15 and 35 by Mantel-Cox test. Analysis is of total data obtained from 12 independent cohorts of mice. 
both intramuscular (i.m.) and intra-nasal (i.n.) modes of vaccination. Two-week-old mice were immunized with heatinactivated PR8 virus $\left(5 \times 10^{5}\right.$ EIU/mouse $)$ through i.m. or i.n. administration. As a control, we also included a group of infant mice that received no immunization. Three weeks postimmunization, mice were challenged with a lethal dose of influenza PR8 virus. The mice were observed for weight loss and clinical signs of severe illness as parameters to determine mortality. Mortality in mice immunized by i.m. administration was comparable to the mortality of mice that received no immunization (Figure 2), indicating that i.m. immunization does not provide protection from a lethal dose of PR8 virus. In contrast, relative to i.m. immunized and unimmunized mice, those mice immunized through i.n. administration of the same inactive PR8 influenza virus have better survival to the infection with the lethal dose of influenza virus (Figure 2). Thus, i.n. administration of the inactive virus provides superior protection in infant mice to influenza virus infection.

\section{Gene Expression Profile Analysis Reveals the Attenuation of IL-6 Gene Expression in Lung Epithelial Cells for Infant Relative to Adult Mice}

The increased protection obtained through an intranasal administration of iPR8 relative to the lack of protection with the intramuscular administration suggests that there is some specific component in the lung which supports the immune response. In addition, the fact that infants are more susceptible than adult mice to primary influenza infection (Figure 1), suggested that there may be tissue specific differences, the adaptive immune response notwithstanding, that are critical for resolution of infection in infant as compared to older mice. Lung epithelial cells are capable of producing a number of cytokines in response to different insults (e.g. viral infection)
(19). To investigate the presence of potential underlining differences between infant and adult lung epithelial cells we performed microarray analyses. We used RNA from lung epithelial cells freshly isolated from naïve infant (2 weeks-old mice) and adult (8 weeks-old mice) mouse lungs. Each pup sample (eight total, four male and four female) included pooled lung epithelial cell RNA preparation from 3 pups, while each adult sample (eight total, four male and four female) contained RNA from a single adult.

Analyses of the microarray results revealed that the number of probe sets that pass an FDR of 0.05 as being differentially expressed in infant versus adult epithelial cells was 7,334. Those passing a binary filter (FDR $<0.05$ and $2 x$ fold change) were 724 . Gene Set Enrichment Analysis and the KEGG database were used to determine pathways that were significantly differently expressed in infant versus adult cells using a criterion of p-value $(<0.05)$ and NES $(>|1.5|)$.

We focused further attention on those immune-related pathways that might be relevant to influenza and that were significantly of lower expression in infant cells as compared to adult. Several pathways were identified. One of the pathways markedly ( $\mathrm{p}=0.0004)$ lower in infants was the "cytosolic DNAsensing pathway" that includes host genes involved in sensing bacteria and viruses such as members of the inflammasome pathway, RIG pathway, type I IFN, chemokines, NF-kB and some cytokines (Figure 3A and Supplementary Figure S6A). Withinpathway analysis defined a cluster of genes with lower expression in epithelial cells from the lungs of infants relative to adult mice (Figure 3A, Supplementary Figure S6A). Interestingly, IL-6 was the gene most significantly lower in lung epithelial cells from infants (Figure 3A, Supplementary Figure S6A). Upon binding to its receptor, IL-6 activates the Jak/Stat pathway leading to the activation of Stat3 (51). The KEGG "Jak/Stat pathway" was also substantially under-expressed in lung epithelial cells from infants relative to adult mice (Figure 3B, Supplementary Figure S6B). This

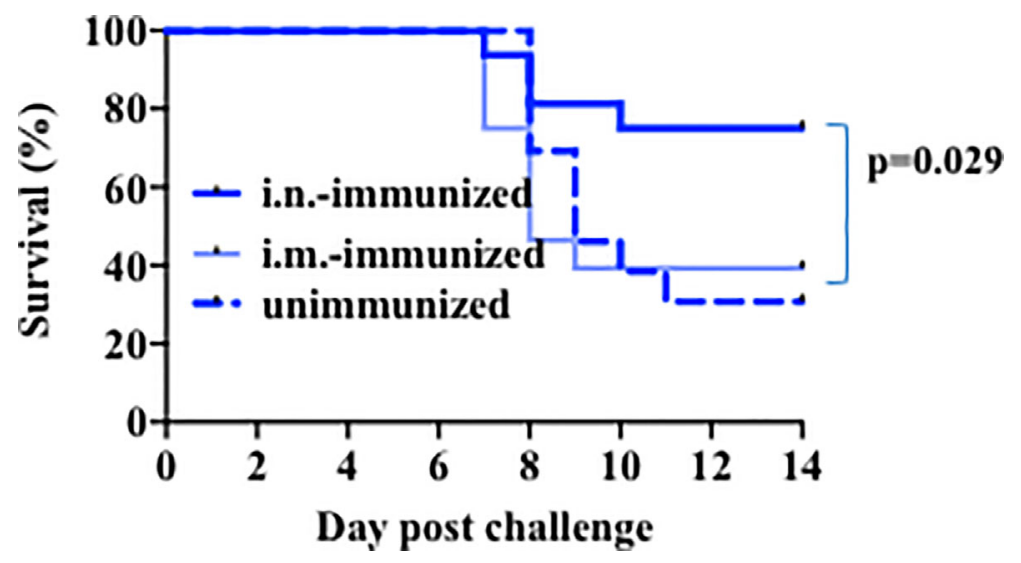

FIGURE 2 | Intranasal immunization with heat inactivated virus protects infant mice against lethal challenge. Mice aged 14-17 days of life were immunized intranasally (Bold Dk blue, $n=16$ ) or intramuscularly (Lt blue, $n=28$ ) with $5 \times 10^{5}$ ElU heat inactivated PR8 virus, or left unimmunized (dotted lines, $n=13$ ). Three weeks later, all mice received a lethal dose of live virus. Y axis: Survival. Mantel-Cox (log-rank) analysis was used to compare unimmunized to i.n.-immunized mice. Significance was set at $\mathrm{p}<0.05$. Analysis is of total data obtained from 8 independent cohorts of mice receiving immunization and or challenge. 
A

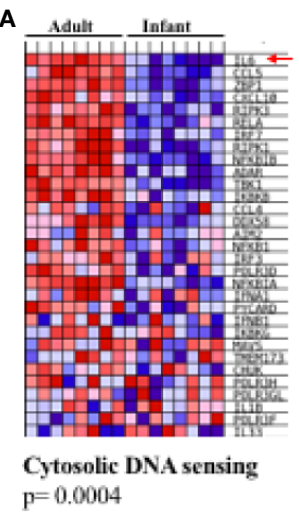

B

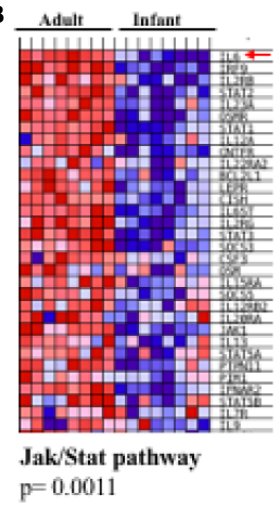

c

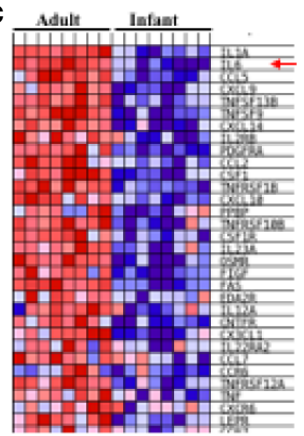

Cytokine/ Cytokine Receptor $\mathrm{p}=0.015$
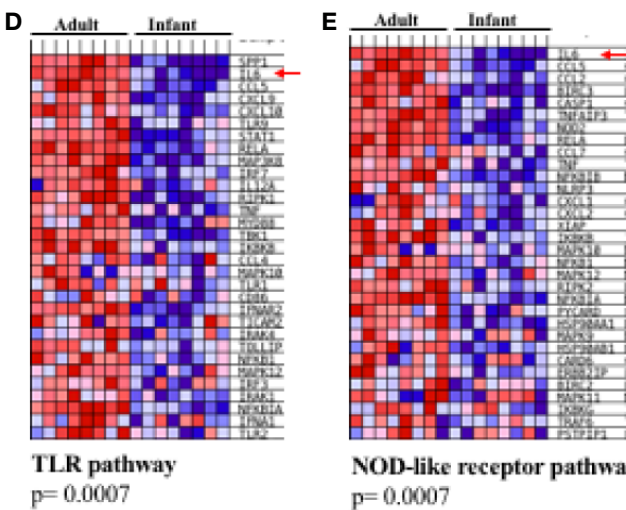

NOD-like receptor pathway $\mathrm{p}=0.0007$

$\mathbf{F}$

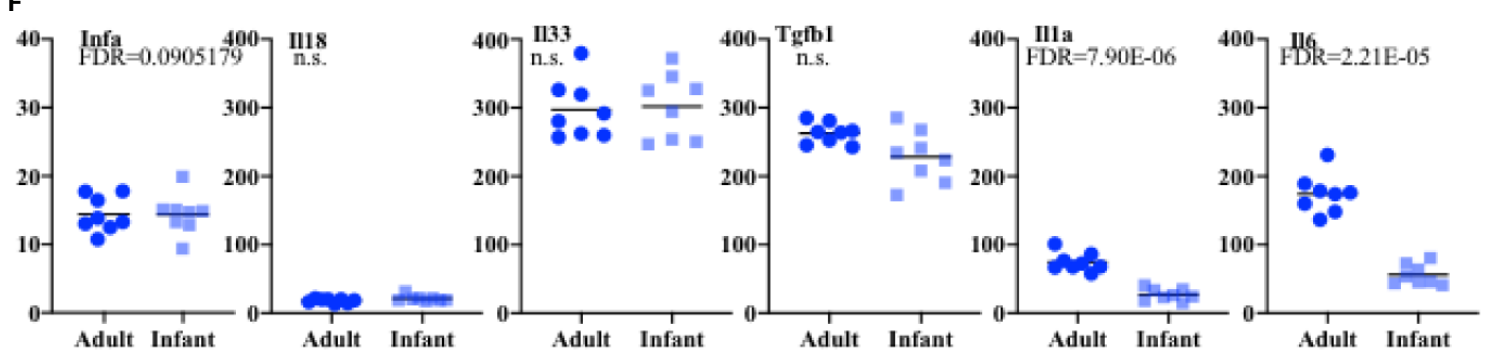

FIGURE 3 | Expression profiling in epithelial cells from infant and adult mice reveals IL-6 deficiency at baseline. Four samples each consisting of pooled epithelial cells from the lungs of three male pups or three female pups aged 14 days (total eight pups samples) or epithelial cells from four individual male and four female adult (8 weeks) lungs (eight adult samples total) were used to isolate RNA and examined by array transcriptome profiling. Gene Set Enrichment Analysis (eight total pools per group) revealed significant differences in expression in pathways relevant to innate immunity. (A-E) Shown are heat maps for the most highly differentially expressed genes within in the various pathways. Red-up regulated, blue down regulated. Red Arrows: IL-6. $P$ values for the gene sets were computed by permuting the gene sets 1,000 times. (F) Transformed RMA data for specific genes in infant versus adult epithelial cell pools. Each symbol refers to a pool of 3 mice. FDR, fold discovery rate (reflecting comparison of groups of pools with modification for multiple comparison testing as part of the array analysis) was calculated using Partek Suites Genomics ${ }^{\circledR} 6.6$ Beta Analysis.

pathway includes genes for cytokines, cytokine receptors, transcription factors, kinases etc. Among them, IL-6 was on the top of the cluster of genes that were significantly lower in epithelial cells from infants (Figure 3B, Supplementary Figure S6B). The "Cytokine-cytokine receptor interaction" pathway, containing a number of genes for cytokines/chemokines and their receptors, was also observed to be significantly decreased and IL-6 together with IL-1 were the genes with the lowest expression in infant lung epithelial cells relative to the expression found in adult mice (Figure 3C, Supplementary Figure S6C). Two other innate immune pathways were identified to be significantly lower in infants. One was the "TLR signaling pathway" that includes pattern recognition receptors responsible for detecting microbial pathogens and generating innate immune responses including molecules such as type I IFN, NF-kB, and inflammatory cytokines (Figure 3D, Supplementary Figure S6D). Another was the NOD-like receptor (NLR) signaling pathway that includes intracellular NLR family members, cytokines regulated by this pathway, caspases, NF$\mathrm{kB}$, and others (Figure 3E, Supplementary Figure S6E). Of interest, among all the different genes included in these two pathways, IL-6 was identified as the gene most reduced in lung epithelial cells in infants (Figures 3D, E, Supplementary Figures S6D, E). Additional comparative modeling for specific cytokines known to be produced by lung epithelial cells further demonstrated selectively reduced expression of IL-6 in infant cells (Figure 3F). We did not observe significant differences in the expression of innate cytokine genes well-known to be expressed in epithelial cells (e.g., IFNa, IFNb, IL-33, IL-18, Figures 3C, F, Supplementary Figure S6C). We have previously shown a constitutive expression of IL-6 (high levels of IL-6 mRNA) in lung epithelial cells isolated from adult wildtype mice under physiological conditions prior to any exposure or insult (38), while no expression was detected in resident leukocytes $\left(\mathrm{CD}^{+} 5^{+}\right.$cells) (38). The results here show that IL-6 gene expression in lung epithelial cells is regulated during development and its expression is attenuated in infants.

\section{Protection of Infant Mice by Intranasal Immunization With Inactive Influenza Virus Requires IL-6}

Administration of IL-6 has been shown to enhance the effectiveness of a subcutaneous inactive influenza virus vaccine in adult mice (40). IL-6 derived from lung epithelial cells could therefore contribute to the protective effect of intranasal vaccines. Since the basal levels of IL-6 gene expression in lung epithelial cells in infants was significantly lower when compared to adults, we examined whether i.n. administration of the 
inactive influenza virus vaccine could upregulate IL-6 expression. Two-week-old mice were administered with an i.n. dose of inactive PR8 virus and the lung was harvested 2 days postimmunization for cytokine expression. Relative to the levels in lung from non-immunized mice, higher levels of IL-6 expression were present in lungs from i.n.-immunized mice (Figure 4A). In contrast, we did not observe an increase in other cytokines, such as TGF $\beta$, in lungs from immunized infants (Figure 4B). Thus, i.n. administration of an inactive influenza virus selectively induced IL-6 expression, suggesting that local production of IL- 6 in the lung could contribute to the protective effect of i.n. administration relative to i.m. administration.

We next investigated the contribution of IL- 6 to the protective effect of i.n. immunization with inactive influenza. IL-6-deficient infants ( 2 weeks-old) were i.n.-immunized with inactive PR8 virus as described above for wildtype mice, or mice were left with no immunization. Three weeks post-immunization, both immunized and non-immunized mice were challenged with a lethal dose of PR8 virus. Intranasal immunization in IL-6-deficient mice resulted in mortality similar to that observed in unimmunized mice (Figures 4C, D) with more than $50 \%$ of mice dying by 10 days after challenge. This was true for both females (Figure 4C) and males (Figure 4D). In contrast to WT female mice (Figure 2) and WT male mice (Figure 4D), i.n. administration of inactive PR8 fails to provide protection in IL-6 KO mice, suggesting that the success of an i.n. immunization with inactive virus relies significantly on IL-6 production in the lung.

\section{Lack of IL-6 Does Not Cause an Exuberant Systemic Immune Response to Influenza Virus Infection}

The results above show that i.n.-immunized IL-6 KO infants experience significant mortality in response to a lethal dose of PR8 influenza virus, while immunized WT infants are significantly protected. The ultimate increased mortality in IL-6-defficient mice could be due to an early-post-challenge exuberant and dysregulated innate response, leading to systemic tissue damage and death. Conversely, our findings could be explained by an insufficiency in the adaptive and protective immune response generated by immunization. To attempt to differentiate between these two possibilities, we investigated the systemic and local immune responses after viral challenge. WT and IL-6 KO mice were intranasally immunized with an inactive PR8 virus as described above. Three weeks post-immunization, mice were infected with a lethal dose of PR8 virus and lungs and serum were harvested 6 days post-infection. Lung viral loads in WT and IL6KO mice were similar (Supplementary Figure S4), suggesting that there were not significant differences in the initial ability to undergo infectious challenge. The levels of soluble ICAM in serum, a marker of systemic inflammatory response, endothelial

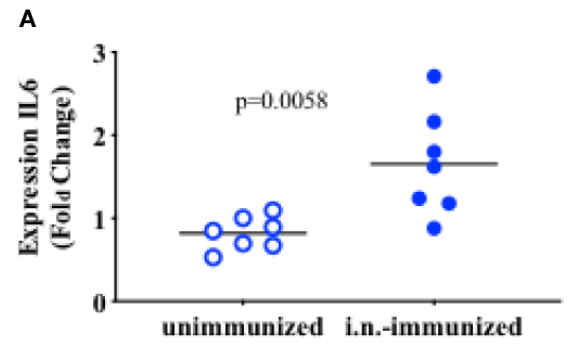

C

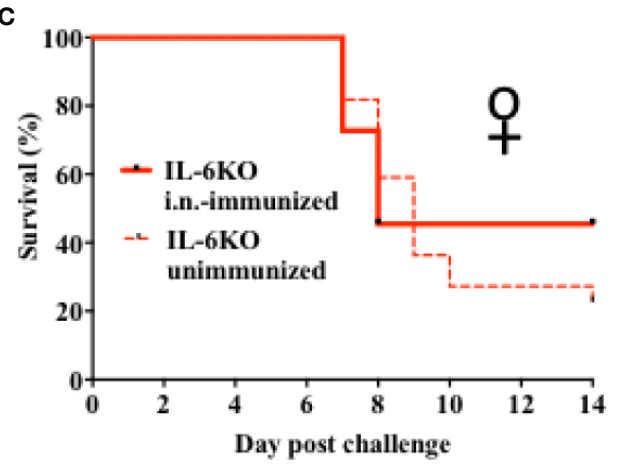

B
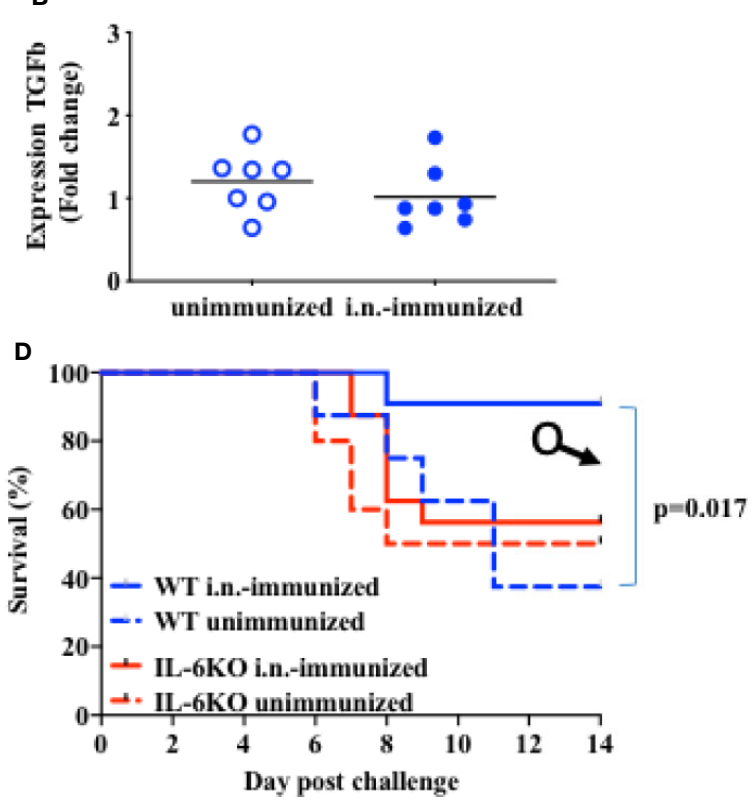

FIGURE 4 | IL-6 is necessary for protective response to an i.n. administration of heat inactivated influenza virus. (A, B): On day 16 of life mice received $5 \times 10^{5}$ EIU of heat-inactivated virus intranasally (DK blue) or not ( $\mathrm{Lt}$ blue). At 2 days after immunization, lungs were harvested and whole lung was used to extract RNA that was later assayed for RNA expression of IL-6 (A) and for TGFb (B) by QPCR. Each symbol represents one mouse. Shown is fold-change relative to unimmunized mice in a single experiment. The t-test was used to test for significant differences between groups. (C, D): Mice aged 14-17 days of life were i.n.-immunized (bold) with $5 \times$ $10^{5}$ EIU heat inactivated virus or left unimmunized (dashed). Three weeks later, all mice received a lethal dose of live virus. Y Axis, survival. $X$ axis, day post challenge. (C) IL-6KO Female mice; i.n.-immunized, $n=11$; unimmunized, $n=22$. (D) Male mice; IL-6KO (red) i.n.-immunized, $n=16$; unimmunized, $n=10$. WT males (blue) i.n. immunized $n=11$, unimmunized, $n=8$ ). Shown is Mantel-Cox (log-rank) analysis of total data using \% initial weight as noted in methods from 5 independent cohorts of mice receiving immunization and or challenge. Significance was set at $p<0.05$. 
cell activation and acute respiratory distress (52, 53), were comparable between WT and IL-6 KO mice (Figure 5A). In addition, there was no difference in weight loss between WT and IL-6 KO mice at this early time point of the infection (Figure 5B). Since it has been reported that IL- 6 deficiency could affect macrophage recruitment by affecting chemokine expression (54), we examined CCL2, important for monocyte trafficking, but no difference was found between WT and IL-6 KO mice (Figure 5C). Further, analysis of cytokine expression in the lungs revealed no difference in inflammatory markers such TNF (Figure 5D). However, the analysis of IFN $\gamma$, a product of the adaptive immune response (CD4 and CD8 T cells), showed a trend toward reduced expression in immunized IL-6 KO mice after the lethal infection with influenza virus (Figure 5E). Thus, these results suggest that the death of immunized IL- 6 KO infants in response to influenza virus is not caused by an enhanced early pathogenic and dysregulated innate immune response, but they instead suggest an impaired memory $\mathrm{T}$ cell response.

\section{IL-6 Is Required for Intranasal Immunization With Inactive Influenza Virus to Sustain Memory T Cell Response in Infant Mice}

To investigate whether, in infants, IL-6 is required for an intranasal vaccine to trigger an efficient adaptive memory immune response, 2-week-old WT and IL-6 KO mice were immunized intranasally with inactive influenza virus as described above. Three weeks post-immunization, lungs from immunized mice were harvested and processed, and different $\mathrm{T}$ cell populations in lung cell homogenate were examined by flow cytometry analysis. Leukocytes were first gated from other cell populations in the lung using CD45 as a pan leukocyte marker (Supplementary Figure S5). We examined the presence of CD8 and CD4 cells within the CD45 cell population. No significant difference in the percentage of CD8 cells could be detected between WT and IL-6 KO i.n.-immunized mice (Figure 6A). Similarly, no significant difference in the presence of CD4 cells was found between WT and IL-6 KO mice (Figure 6B). However, when we examined the presence of memory CD8 cells using CD44 ${ }^{\text {high }}$ as marker, the frequency of memory cells was markedly reduced in lungs from immunized IL-6 KO infants relative to WT infants (Figure 6C). Similar results were obtained for CD4 cells. The frequency of CD4 CD $44^{\text {high }}$ cells was significantly lower in IL-6 KO infants relative to WT mice (Figure 6D). Thus, during intranasal influenza immunization of infants, IL-6 does not seem to promote the recruitment of lymphocytes, but is important in a sustained memory $\mathrm{T}$ cell response.

IL-6 has been shown to promote antibody production indirectly by acting on CD8 and CD4 T cells and supporting their IL-21 production. IL-21 in turn acts on B cells to promote
A

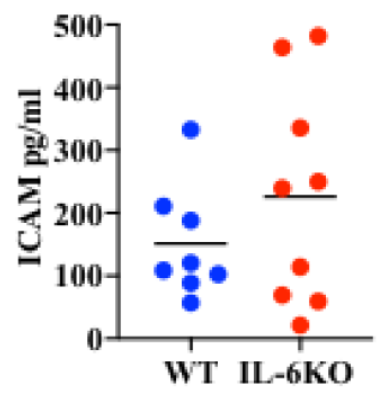

C

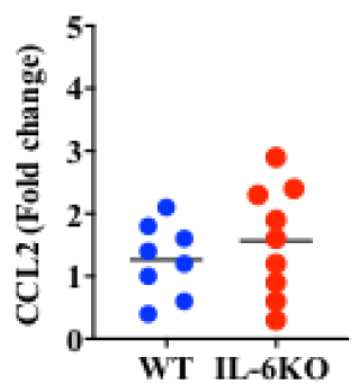

B

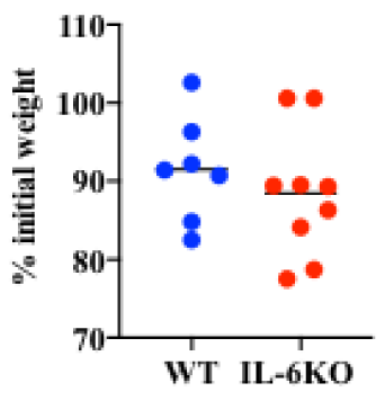

D

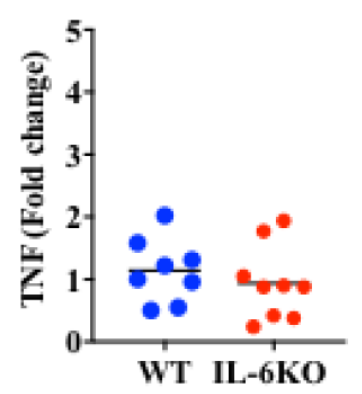

E

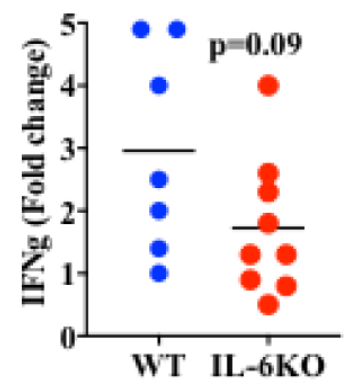

FIGURE 5 | Response to a lethal dose of influenza in i.n. immunized WT and IL-6KO mice. Mice received intranasal immunization with inactive virus on day 14 of life. Three weeks later, mice were weighed and given a lethal dose PR8 virus. Six days after challenge mice were euthanized and tissues harvested for analysis. (A) Serum ICAM was analyzed by Luminex ${ }^{T M}$. (B) Weight of mice, 6 days post-infection. Shown is the \% relative to the initial weight prior to infection. (C-E) Whole lung relative mRNA expression for CCL2 (C), TNF (D) and IFNg (E) determined by real time RT-PCR. Values show fold induction relative to a WT. Each dot represents a mouse. One experiment is shown. Bars indicate mean for group. Comparison utilized the t test, with significance set at $\mathrm{p}<0.05$. 
A

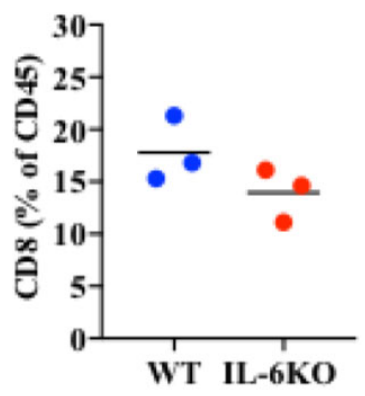

C

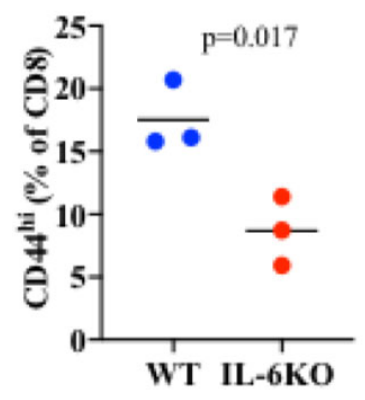

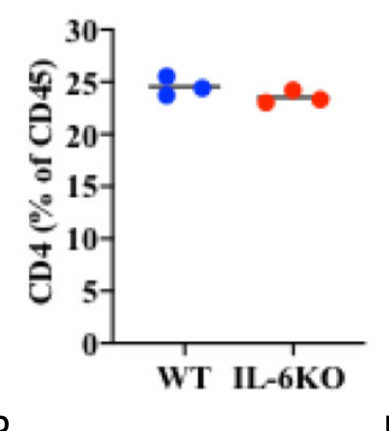

E

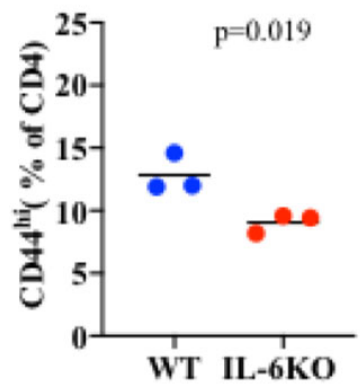

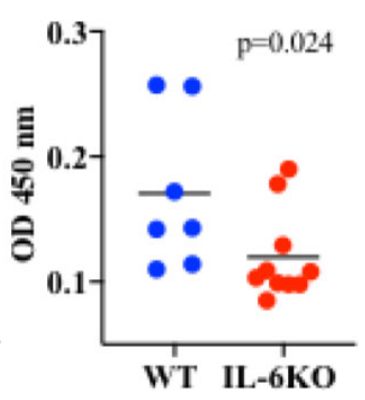

FIGURE 6 | Lower frequency of memory CD8 and CD4 cells in the lungs of i.n. immunized IL-6 KO mice. Mice $(n=3)$ received intranasal immunization with inactive virus on day 14 of life. Three weeks later, lungs were extracted and analyzed by flow cytometry. (A) Cells were gated for CD45 first. \% of CD8 cells within the CD45+ population is showed. (B) Cells were gated for CD45 first. \% of CD4 cells within the CD45+ population is showed. (C) Cells were gated for CD45 first, and then for CD8 cells. \% of CD44 high within CD8 cells is shown. (D) Cells were gated for CD45 first, and then for CD4 cells. \% of CD44 high within CD4 cells is shown. $p$ value is determined by $t$ test. (E) PR8-specific IgG antibody titer in serum determined by ELISA. $p$ value is calculated using the Mann-Whitney test, with significance set at $p$ $<0.05$. One experiment is shown.

antibody production, primarily IgG $(40,55)$. We therefore also examined the presence of influenza-specific IgG in the serum of WT and IL-6 KO infants 3 weeks post-immunization. The levels of PR8-specific IgG were reduced in immunized IL-6 KO infants compared with WT mice (Figure 6E). Thus, during i.n. immunization of infants, IL-6 plays an important role in sustaining effective $\mathrm{T}$ and $\mathrm{B}$ cell adaptive memory.

\section{DISCUSSION}

Infant immunity remains an important area of focus due to significant existing morbidity related to infectious disease. Influenza infection remains a major challenge among infections in children under five years of age (56). While historically it is believed to be due to an inappropriate T/B cell immune response, pathology and resolution of primary influenza virus infection is not dependent on the adaptive immunity but requires innate immunity (57). Another potential difference between children and adults is dose of the virus to which they are environmentally exposed relative to lung size (again, determined by body size). However, in our studies here we show that even when influenza viral dose is normalized to body weight (reflecting lung size), infant mice are more susceptible to sublethal dose than adult mice. Therefore, an increased virus dose/lung size ratio does not seem to be the main cause for the enhanced sensitivity to influenza virus response in infants. This disparity seems to be a developmental issue. Here, our Microarray studies have revealed a different gene expression profile in epithelial cells from the lungs of adult and infant mice prior to exposure to any infection or other type of insult.

The view that infant immune cells are inherently deficient contrasts with the view that, given the appropriate environment infant immune cells can behave similarly to adult $(11,13-15,58)$. While it is generally believed that the deficient response of very young children to influenza infection is due to antigen inexperience in $\mathrm{T}$ or $\mathrm{B}$ cells, the difference in the immune response could also be determined by cells other than immune cells that contribute to shape the adaptive immune response mediated by $\mathrm{T}$ or $\mathrm{B}$ cells.

For instance, epithelial cells (as well as endothelial cells) can produce cytokines and other factors that can modulate the type or strength of CD4 and CD8 cell mediated immune responses. Lung epithelial cells are the main cell target of influenza virus, as they express high levels of sialic acid on the cell surface to which influenza binds, subsequently enters, and replicates (19). However, lung epithelial cells can also orchestrate the innate anti-viral immune response. Influenza virus replication induces expression and production of type I IFN that acts as an anti- 
viral factor (39). Furthermore, lung epithelial cells are known to be able to produce different inflammatory cytokines (e.g. IL-33, IL-1, IL-6) that can then have an effect on the adaptive immune response (19). Under physiologic conditions (not during exposure to an insult) lung epithelial cells from adult mice express high levels of IL- 6 mRNA in contrast to the relative absence during of IL- 6 expression in lung resident macrophages (38). IL-6 production and secretion by mouse and human lung epithelial cells is triggered during infection with influenza virus and other viruses such coronavirus $(39,59,60)$. IL-6 plays a pivotal role in dictating the types of CD4 and CD8 cell responses. Further, IL- 6 has been shown to promote differentiation of CD4 cells into Th2 and Tfh cells that can then, by secreting IL-21 and IL-4, enhance isotype switching and antibody production in B cells $(40,61,62)$. In influenza virus infection, IL- 6 triggers IL-21 production by CD 4 cells and IL-21 is essential for virus antibody response (40). In addition, we have also shown that IL-6 makes CD8 cells to become helpers of B cells through induction of IL-21. During influenza virus, CD8 cells in the lung but not in lymph nodes produce IL21 and this effect requires IL-6 (55). Thus, the difference in IL-6 production by lung epithelial cells between infant and adults could account for the difference in $\mathrm{T}$ and $\mathrm{B}$ cell responses to influenza virus in the lung.

In this study, our gene profile analyses revealed marked differences the expression of immune regulators in lung epithelial cells from infants as compared to adult mice. Our pathway analysis has revealed IL- 6 as a highly selective gene that is lower in pre-weanling epithelial cells. Interestingly, expression of other cytokines that play a role in antiviral immune responses (e.g. type I IFN), regulation of the adaptive immune response (e.g. IL-33) or the innate immune response (e.g. IL-18) was not different between infants and adult mice, further highlighting the potential significance of the differential expression of IL- 6 that we observed. The evidence pointing to IL-6 expression in lung epithelial cells as being developmentally regulated could have a major impact on the understanding of childhood immune responses to pathogenic influenza viruses, but also to other respiratory viruses e.g., those producing SARS.

Since our gene expression profiling reveals a marked reduction (more than 3-fold lower) in the IL-6 expression in lung epithelial cells from infants relative to adult mice, it is possible that the lower IL-6 levels in the lungs could be responsible for the increased susceptibility. In this regard, adult mice lacking IL-6 or IL-6R die in response to sublethal dose of influenza virus in part due to the reduced number of neutrophils in the lung to mediate virus clearance (41). In addition, IL-6 can ameliorate acute lung injury in influenza virus infection in mice by promoting tissue repair (63). In humans, the systemic treatment with tocilizumab, the blocking anti-IL-6R antibody approved for treatment of rheumatoid arthritis, has also been shown to increase the risk to respiratory virus infection (64). Therefore, although exaggerated levels of IL- 6 have been observed in adult patients with acute respiratory distress syndrome due to massive lung tissue damage $(65,66)$, IL-6 also likely provides protection from infections with influenza virus and other respiratory viruses (66).

Current influenza vaccination formulations include a live attenuated virus given intranasally, and an inactivated virus administered via intramuscular injection (26). Each has variable protection, depending on the formulation, the year of production, and the population vaccinated (27). In addition, the live-attenuated vaccine is not approved for very young children less than 2 years of age. Thus, this population remains at high risk every season. Here we show that a vaccine with inactive influenza virus provides protection to $\sim 15$ day-old mice when administered intranasally, suggesting a potential alternative option of vaccination for this highly susceptible population of infant children. Interestingly, our data also show that the protective effect of the intranasal immunization with the inactive influenza virus in infant mice is dependent on IL-6 since it fails to provide protection in IL- 6 deficient mice. In contrast to i.n. administration, intramuscular administration of the inactive influenza virus did not provide protection in infant mice, stressing the importance of the intranasal route over the intramuscular route at this dose. The induction of IL- 6 by the i.n. vaccine with the inactive virus suggested that this IL-6 may come from lung epithelial cells, although future studies will be needed to further demonstrate that this is the case. Using a commercially formulated multivalent vaccine for the $2012 / 2013$ season, it has been reported that i.m. vaccination produced a protective response in infant mice (67). It is possible that the mix of influenza virus strains $(\mathrm{H} 3 \mathrm{~N} 2$ in addition to $\mathrm{H} 1 \mathrm{~N} 1)$ in that particular seasonal vaccine could trigger a stronger immune response. However, correlating with our studies in infants, i.m. administration of inactive influenza virus in adult mice also induces a limited antibody response (40). The superior efficacy of the inactive virus vaccine when administered i.n. further reinforces the relevance of the environment where the immune response takes place. Most approved antiviral vaccines are based on their ability to induce antibody production. There is evidence that LAIV may trigger some T cell mediated protection $(68,69)$ although it has not been fully demonstrated. Intriguingly, here we show a reduction in the frequency of activated CD4 and CD8 cells in the lung in immunized IL- 6 deficient mice relative to WT mice. Thus, it is possible that the lower efficacy of our inactive influenza viral vaccine in the IL-6 deficient mice could be due to impaired memory CD4/CD8 $\mathrm{T}$ cell response and the subsequent antibody response.

This investigation posits that vaccination of the infant can generate a protective response against lethal challenge if the correct environment is achieved. Our studies are in keeping with previous studies suggesting that immunization of infants against differing antigens, depending on the right source and format can lead to $\mathrm{T}$ cell activation in the relevant tissue and/or systemic antibody production (70). The critical role played by IL- 6 in these studies when taken in context are consistent with the idea that environment matters in the development of infant immunity. Further understanding and the ability to harness specific tissue environments may aid in developing strategies for protection of the very young. 


\section{DATA AVAILABILITY STATEMENT}

The raw data supporting the conclusions of this article will be made available by the authors, without undue reservation.

\section{ETHICS STATEMENT}

The animal study was reviewed and approved by University of Vermont Institutional Animal Care and Use Committee.

\section{AUTHOR CONTRIBUTIONS}

EB designed and helped perform experiments, managed, analyzed, and interpreted the data, and wrote/edited the figures and manuscript. JK helped do gene expression analysis studies. BT helped perform experiments, as did KP and KK. LH provided PR8 and helped with data analysis and editing. MR helped perform experiments, analyzed, and interpreted the data, and wrote and edited the manuscript. All authors contributed to the article and approved the submitted version.

\section{FUNDING}

Support for this work came from NIH P30GM118228 to the Vermont Center for Immunology and Infectious Disease, P30GM103532 to the Vermont Lung Center, the University of

\section{REFERENCES}

1. Scheltema NM, Gentile A, Lucion F, Nokes DJ, Munywoki PK, Madhi SA, et al. Global respiratory syncytial virus-associated mortality in young children (RSV GOLD): a retrospective case series. Lancet Glob Health (2017) 5:e98491. doi: 10.1016/s2214-109x(17)30344-3

2. Liao X, Hu Z, Liu W, Lu Y, Chen D, Chen M, et al. New Epidemiological and Clinical Signatures of 18 Pathogens from Respiratory Tract Infections Based on a 5-Year Study. PloS One (2015) 10:e0138684. doi: 10.1371/journal.pone. 0138684

3. Blanton L, Dugan VG, Abd Elal AI, Alabi N, Barnes J, Brammer L, et al. Update: Influenza Activity - United States, September 30, 2018-February 2, 2019. MMWR Morb Mortal Wkly Rep (2019) 68:125-34. doi: 10.15585/ mmwr.mm6806al

4. Fike AJ, Kumova OK, Tardif VJ, Carey AJ. Neonatal influenza-specific effector CTLs retain elevated CD31 levels at the site of infection and have decreased IFN-gamma production. J Leukoc Biol (2019) 105:539-49. doi: 10.1002/jlb.4a0518-191r

5. Lederberg J. Genes and antibodies. Sci (New York NY) (1959) 129:1649-53. doi: $10.1126 /$ science.129.3364.1649

6. Mold JE, Michaelsson J, Burt TD, Muench MO, Beckerman KP, Busch MP, et al. Maternal alloantigens promote the development of tolerogenic fetal regulatory T cells in utero. Sci (New York NY) (2008) 322:1562-5. doi: $10.1126 /$ science.1164511

7. Ohtsuka Y. Food intolerance and mucosal inflammation. Pediatr Int (2015) 57:22-9. doi: 10.1111/ped.12546

8. Mold JE, Venkatasubrahmanyam S, Burt TD, Michaelsson J, Rivera JM, Galkina SA, et al. Fetal and adult hematopoietic stem cells give rise to distinct T cell lineages in humans. Sci (New York NY) (2010) 330:1695-9. doi: 10.1126/science.1196509
Vermont College of Medicine New Research Initiative Program, the UVM Flow Cytometry and Cell Sorting Facility, and the Department of Obstetrics, Gynecology and Reproductive Sciences. MR was also supported by NIH R56 AI116255 and R01 AI051454. LH and BT are supported by the University of Connecticut Center on Aging. JK is supported by the University of Colorado Cancer Center Bioinformatics/Biostatistics Shared Resources, supported by NIH P30CA046934. Research reported herein was also supported by an Institutional Development Award (IDeA) from the National Institute of General Medical Sciences of the National Institutes of Health under grant number P20GM103449, The Vermont Genetics Network.

\section{ACKNOWLEDGMENTS}

We are grateful for discussions with Matt Poynter and Cory Teuscher in the Vermont Center for Immunology and Infectious Disease, and with James DeGregori, Department of Biochemistry and Molecular Genetics, University of Colorado Anschutz Medical Campus. Thanks goes to Emily Giddings for technical support.

\section{SUPPLEMENTARY MATERIAL}

The Supplementary Material for this article can be found online at: https://www.frontiersin.org/articles/10.3389/fimmu.2020. 568978/full\#supplementary-material

9. Ridge JP, Fuchs EJ, Matzinger P. Neonatal tolerance revisited: turning on newborn T cells with dendritic cells. [see comment]. Sci (New York NY) (1996) 271:1723-6. doi: 10.1126/science.271.5256.1723

10. Matzinger P. Friendly and dangerous signals: is the tissue in control? Nat Immunol (2007) 8:11-3. doi: 10.1038/ni0107-11

11. Fadel SA, Cowell LG, Cao S, Ozaki DA, Kepler TB, Steeber DA, et al. Neonateprimed CD8+ memory cells rival adult-primed memory cells in antigendriven expansion and anti-viral protection. Int Immunol (2006) 18:249-57. doi: 10.1093/intimm/dxh360

12. Sinnott BD, Park B, Boer MC, Lewinsohn DA, Lancioni CL. Direct TLR2 Costimulation Unmasks the Proinflammatory Potential of Neonatal CD4+ T Cells. J Immunol (2016) 197:68-77. doi: 10.4049/jimmunol.1501297

13. Forsthuber T, Yip HC, Lehmann PV. Induction of TH1 and TH2 immunity in neonatal mice. Sci (New York NY) (1997) 271:1728-30. doi: 10.1126/ science.271.5256.1728

14. Arulanandam BP, Mittler JN, Lee WT, O’Toole M, Metzger DW. Neonatal administration of IL-12 enhances the protective efficacy of antiviral vaccines. J Immunol (2000) 164:3698-704. doi: 10.4049/jimmunol.164.7.3698

15. Kollmann TR, Kampmann B, Mazmanian SK, Marchant A, Levy O. Protecting the Newborn and Young Infant from Infectious Diseases: Lessons from Immune Ontogeny. Immunity (2017) 46:350-63. doi: 10.1016/j.immuni.2017.03.009

16. Huygens A, Lecomte S, Tackoen M, Olislagers V, Delmarcelle Y, Burny W, et al. Functional Exhaustion Limits CD4+ and CD8+ T-Cell Responses to Congenital Cytomegalovirus Infection. J Infect Dis (2015) 212:484-94. doi: 10.1093/infdis/jiv071

17. Debock I, Flamand V. Unbalanced Neonatal CD4(+) T-Cell Immunity. Front Immunol (2014) 5:393. doi: 10.3389/fimmu.2014.00393

18. Cohn M. Giving Context to Non-self-marker Theories of Immune Responsiveness. Scand J Immunol (2017) 86:124-9. doi: 10.1111/sji.12579 
19. Denney L, Ho L-P. The role of respiratory epithelium in host defence against influenza virus infection. BioMed J (2018) 41:218-33. doi: 10.1016/ j.bj.2018.08.004

20. Chilvers MA, Rutman A, O'Callaghan C. Functional analysis of cilia and ciliated epithelial ultrastructure in healthy children and young adults. Thorax (2003) 58:333-8. doi: 10.1136/thorax.58.4.333

21. Smith LS, Zimmerman JJ, Martin TR. Mechanisms of acute respiratory distress syndrome in children and adults: a review and suggestions for future research. Pediatr Crit Care Med (2013) 14:631-43. doi: 10.1097/ PCC.0b013e318291753f

22. Mariani TJ. Update on Molecular Biology of Lung DevelopmentTranscriptomics. Clin Perinatol (2015) 42:685-95. doi: 10.1016/ j.clp.2015.08.001

23. Wang Q, Bhattacharya S, Mereness JA, Anderson C, Lillis JA, Misra RS, et al. A novel in vitro model of primary human pediatric lung epithelial cells. Pediatr Res (2020) 87:511-7. doi: 10.1038/s41390-019-0340-9

24. Clay CC, Reader JR, Gerriets JE, Wang TT, Harrod KS, Miller LA. Enhanced viral replication and modulated innate immune responses in infant airway epithelium following H1N1 infection. J Virol (2014) 88:7412-25. doi: 10.1128/jvi.00188-14

25. Agarkhedkar S, Chhatwal J, Kompithra RZ, Lalwani SK, Narayan A, Muninarayanaswam $\mathrm{V}$, et al. Immunogenicity and safety of an intramuscular split-virion quadrivalent inactivated influenza vaccine in individuals aged $>/=6$ months in India. Hum Vaccines Immunother (2019) 15:973-7. doi: 10.1080/ 21645515.2019.1565259

26. Valdin HL, Begue RE. Influenza vaccines effectiveness 2013-14 through 201516, a test-negative study in children. Vaccine (2017) 35:4088-93. doi: 10.1016/ j.vaccine.2017.06.050

27. Grohskopf LA, Sokolow LZ, Broder KR, Walter EB, Fry AM, Jernigan DB. Prevention and Control of Seasonal Influenza with Vaccines: Recommendations of the Advisory Committee on Immunization PracticesUnited States, 2018-19 Influenza Season. MMWR Recomm Rep (2018) 67:120. doi: 10.15585/mmwr.rr6703a1

28. COMMITTEE ON INFECTIOUS DISEASES. Recommendations for Prevention and Control of Influenza in Children, 2019-2020. Pediatrics (2019) 144(4):e20192478. doi: 10.1542/peds.2019-2478

29. Bergen R, Black S, Shinefield H, Lewis E, Ray P, Hansen J, et al. Safety of coldadapted live attenuated influenza vaccine in a large cohort of children and adolescents. Pediatr Infect Dis J (2004) 23:138-44. doi: 10.1097/ 01.inf.0000109392.96411.4f

30. Belshe RB, Edwards KM, Vesikari T, Black SV, Walker RE, Hultquist M, et al. Live attenuated versus inactivated influenza vaccine in infants and young children. N Engl J Med (2007) 356:685-96. doi: 10.1056/ NEJMoa065368

31. Caspard H, Steffey A, Mallory RM, Ambrose CS. Evaluation of the safety of live attenuated influenza vaccine (LAIV) in children and adolescents with asthma and high-risk conditions: a population-based prospective cohort study conducted in England with the Clinical Practice Research Datalink. BMJ Open (2018) 8:e023118. doi: 10.1136/bmjopen-2018-023118

32. Turner PJ, Fleming L, Saglani S, Southern J, Andrews NJ, Miller E. Safety of live attenuated influenza vaccine (LAIV) in children with moderate to severe asthma. J Allergy Clin Immunol (2020) 145:1157-1164.e1156. doi: 10.1016/ j.jaci.2019.12.010

33. Poehling KA, Caspard H, Peters TR, Belongia EA, Congeni B, Gaglani M, et al. 2015-2016 Vaccine Effectiveness of Live Attenuated and Inactivated Influenza Vaccines in Children in the United States. Clin Infect Dis an Off Publ Infect Dis Soc America (2018) 66:665-72. doi: 10.1093/cid/cix869

34. Mandelboim M, Glatman-Freedman A, Drori Y, Sherbany H, Pando R, Sefty $\mathrm{H}$, et al. Ineffectiveness of the 2014-2015 H3N2 influenza vaccine. Oncotarget (2016) 7:1185-92. doi: 10.18632/oncotarget.6746

35. Grohskopf LA, Sokolow LZ, Fry AM, Walter EB, Jernigan DB. Update: ACIP Recommendations for the Use of Quadrivalent Live Attenuated Influenza Vaccine (LAIV4) - United States, 2018-19 Influenza Season. MMWR Morb Mortal Wkly Rep (2018) 67:643-5. doi: 10.15585/mmwr.mm6722a5

36. Rincon M. Interleukin-6: from an inflammatory marker to a target for inflammatory diseases. Trends Immunol (2012) 33:571-7. doi: 10.1016/ j.it.2012.07.003

37. Rose-John S. Interleukin-6 Family Cytokines. Cold Spring Harb Perspect Biol (2018) 10. doi: 10.1101/cshperspect.a028415
38. Neveu WA, Bernardo E, Allard JL, Nagaleekar V, Wargo MJ, Davis RJ, et al. Fungal Allergen \{beta\}-glucans Trigger p38 MAPK-mediated IL-6 Translation in Lung Epithelial Cells. Am J Respir Cell Mol Biol (2011) 45(6):1133-41. doi: $10.1165 / \mathrm{rcmb} .2011-0054 \mathrm{OC}$

39. Matsukura S, Kokubu F, Noda H, Tokunaga H, Adachi M. Expression of IL-6, IL-8, and RANTES on human bronchial epithelial cells, NCI-H292, induced by influenza virus A. J Allergy Clin Immunol (1996) 98:1080-7. doi: 10.1016/ s0091-6749(96)80195-3

40. Dienz O, Eaton SM, Bond JP, Neveu W, Moquin D, Noubade R, et al. The induction of antibody production by IL- 6 is indirectly mediated by IL- 21 produced by CD4+ T cells. J Exp Med (2009) 206:69-78. doi: 10.1084/ jem.20081571

41. Dienz O, Rud JG, Eaton SM, Lanthier PA, Burg E, Drew A, et al. Essential role of IL-6 in protection against H1N1 influenza virus by promoting neutrophil survival in the lung. Mucosal Immunol (2012) 5:258-66. doi: 10.1038/ mi.2012.2

42. Yang J, Sakai J, Siddiqui S, Lee RC, Ireland DDC, Verthelyi D, et al. IL-6 Impairs Vaccine Responses in Neonatal Mice. Front Immunol (2018) 9:3049. doi: 10.3389/fimmu.2018.03049

43. Poli V, Balena R, Fattori E, Markatos A, Yamamoto $\mathrm{M}$, Tanaka $\mathrm{H}$, et al. Interleukin- 6 deficient mice are protected from bone loss caused by estrogen depletion. EMBO J (1994) 13:1189-96. doi: 10.1002/j.1460-2075.1994. tb06368.x

44. Scholtissek C, Rott R. Effect of temperature on the multiplication of an Influenza virus. J Gen Virol (1969) 5:283-90. doi: 10.1099/0022-1317-5-2-283

45. Jonges M, Liu WM, van der Vries E, Jacobi R, Pronk I, Boog C, et al. Influenza virus inactivation for studies of antigenicity and phenotypic neuraminidase inhibitor resistance profiling. J Clin Microbiol (2010) 48:928-40. doi: 10.1128/ jcm.02045-09

46. Irizarry RA, Bolstad BM, Collin F, Cope LM, Hobbs B, Speed TP. Summaries of Affymetrix GeneChip probe level data. Nucleic Acids Res (2003) 31:e15. doi: 10.1093/nar/gng015

47. Kanehisa M, Goto S, Furumichi M, Tanabe M, Hirakawa M. KEGG for representation and analysis of molecular networks involving diseases and drugs. Nucleic Acids Res (2010) 38:D355-360. doi: 10.1093/nar/gkp896

48. Subramanian A, Tamayo P, Mootha VK, Mukherjee S, Ebert BL, Gillette MA, et al. Gene set enrichment analysis: a knowledge-based approach for interpreting genome-wide expression profiles. Proc Natl Acad Sci U States America (2005) 102:15545-50. doi: 10.1073/pnas.0506580102

49. Preaud E, Durand L, Macabeo B, Farkas N, Sloesen B, Palache A, et al. Annual public health and economic benefits of seasonal influenza vaccination: a European estimate. BMC Public Health (2014) 14:813. doi: 10.1186/14712458-14-813

50. Shinjoh M, Sugaya N, Yamaguchi Y, Iibuchi N, Kamimaki I, Goto A, et al. Inactivated influenza vaccine effectiveness and an analysis of repeated vaccination for children during the 2016/17 season. Vaccine (2018) 36:5510-8. doi: 10.1016/j.vaccine.2018.07.065

51. Heinrich PC, Behrmann I, Haan S, Hermanns HM, Muller-Newen G, Schaper F. Principles of interleukin (IL)-6-type cytokine signalling and its regulation. Biochem J (2003) 374:1-20. doi: 10.1042/bj20030407

52. Marchesi S, Lupattelli G, Lombardini R, Sensini A, Siepi D, Mannarino M, et al. Acute inflammatory state during influenza infection and endothelial function. Atherosclerosis (2005) 178:345-50. doi: 10.1016/j.atherosclerosis. 2004.08.036

53. Briassoulis G, Papassotiriou I, Mavrikiou M, Lazaropoulou C, Margeli A. Longitudinal course and clinical significance of TGF-beta1, sL- and sESelectins and sICAM-1 levels during severe acute stress in children. Clin Biochem (2007) 40:299-304. doi: 10.1016/j.clinbiochem.2006.11.015

54. Romano M, Sironi M, Toniatti C, Polentarutti N, Fruscella P, Ghezzi P, et al. Role of IL- 6 and its soluble receptor in induction of chemokines and leukocyte recruitment. Immunity (1997) 6:315-25. doi: 10.1016/s1074-7613(00)80334-9

55. Yang R, Masters AR, Fortner KA, Champagne DP, Yanguas-Casas N, Silberger DJ, et al. IL-6 promotes the differentiation of a subset of naive CD8+ T cells into IL-21-producing B helper CD8+ T cells. J Exp Med (2016) 213:2281-91. doi: 10.1084/jem.20160417

56. Misra RS, Nayak JL. The Importance of Vaccinating Children and Pregnant Women against Influenza Virus Infection. Pathogens (2019) 8:265. doi: $10.3390 /$ pathogens 8040265 
57. Eichelberger M, Allan W, Zijlstra M, Jaenisch R, Doherty PC. Clearance of influenza virus respiratory infection in mice lacking class I major histocompatibility complex-restricted CD8+ T cells. J Exp Med (1991) 174:875-80. doi: 10.1084/jem.174.4.875

58. Schwartz DH, Hurwitz JL, Greenspan NS, Doherty PC. Priming of virusimmune memory T cells in newborn mice. Infect Immun (1984) 43:202-5. doi: 10.1128/IAI.43.1.202-205.1984

59. Liu S, Yan R, Chen B, Pan Q, Chen Y, Hong J, et al. Influenza Virus-Induced Robust Expression of SOCS3 Contributes to Excessive Production of IL-6. Front Immunol (2019) 10:1843. doi: 10.3389/fimmu.2019.01843

60. Yoshikawa T, Hill T, Li K, Peters CJ, Tseng CT. Severe acute respiratory syndrome (SARS) coronavirus-induced lung epithelial cytokines exacerbate SARS pathogenesis by modulating intrinsic functions of monocyte-derived macrophages and dendritic cells. J Virol (2009) 83:3039-48. doi: 10.1128/jvi.01792-08

61. Carbone G, Wilson A, Diehl SA, Bunn J, Cooper SM, Rincon M. Interleukin-6 receptor blockade selectively reduces IL-21 production by CD4 T cells and IgG4 autoantibodies in rheumatoid arthritis. Int J Biol Sci (2013) 9:279-88. doi: 10.7150/ijbs.5996

62. Diehl S, Chow CW, Weiss L, Palmetshofer A, Twardzik T, Rounds L, et al. Induction of NFATc2 expression by interleukin 6 promotes T helper type 2 differentiation. J Exp Med (2002) 196:39-49. doi: 10.1084/jem.20020026

63. Yang ML, Wang CT, Yang SJ, Leu CH, Chen SH, Wu CL, et al. IL-6 ameliorates acute lung injury in influenza virus infection. Sci Rep (2017) 7:43829. doi: 10.1038/srep43829

64. Lang VR, Englbrecht M, Rech J, Nüsslein H, Manger K, Schuch F, et al. Risk of infections in rheumatoid arthritis patients treated with tocilizumab. Rheumatol (Oxford) (2012) 51:852-7. doi: 10.1093/rheumatology/ker223

65. Wu W, Shi Y, Gao H, Liang W, Sheng J, Li L. Immune derangement occurs in patients with H7N9 avian influenza. Crit Care (2014) 18:R43. doi: 10.1186/cc13788
66. Velazquez-Salinas L, Verdugo-Rodriguez A, Rodriguez LL, Borca MV. The Role of Interleukin 6 During Viral Infections. Front Microbiol (2019) 10:1057. doi: $10.3389 /$ fmicb.2019.01057

67. McDonald JU, Zhong Z, Groves HT, Tregoning JS. Inflammatory responses to influenza vaccination at the extremes of age. Immunology (2017) 151:451-63. doi: $10.1111 /$ imm. 12742

68. Lartey S, Zhou F, Brokstad KA, Mohn KG, Slettevoll SA, Pathirana RD, et al. Live-Attenuated Influenza Vaccine Induces Tonsillar Follicular T Helper Cell Responses That Correlate With Antibody Induction. J Infect Dis (2020) 221:21-32. doi: 10.1093/infdis/jiz321

69. Mohn KG, Brokstad KA, Islam S, Oftung F, Tøndel C, Aarstad HJ, et al. Early Induction of Cross-Reactive CD8+ T-Cell Responses in Tonsils After LiveAttenuated Influenza Vaccination in Children. J Infect Dis (2020) 221:1528 37. doi: 10.1093/infdis/jiz583

70. Bot A, Bot S, Garcia-Sastre A, Bona C. DNA immunization of newborn mice with a plasmid-expressing nucleoprotein of influenza virus. Viral Immunol (1996) 9:207-10. doi: 10.1089/vim.1996.9.207

Conflict of Interest: The authors declare that the research was conducted in the absence of any commercial or financial relationships that could be construed as a potential conflict of interest.

Copyright (c) 2020 Bonney, Krebs, Kim, Prakash, Torrance, Haynes and Rincon. This is an open-access article distributed under the terms of the Creative Commons Attribution License (CC BY). The use, distribution or reproduction in other forums is permitted, provided the original author(s) and the copyright owner(s) are credited and that the original publication in this journal is cited, in accordance with accepted academic practice. No use, distribution or reproduction is permitted which does not comply with these terms. 